\title{
Modelling the Potential Role of Media Campaigns in Ebola Transmission Dynamics
}

\author{
Sylvie Diane Djiomba Njankou and Farai Nyabadza \\ Department of Mathematical Science, Stellenbosch University, Private Bag X1, Matieland 7600, South Africa \\ Correspondence should be addressed to Farai Nyabadza; f.nyaba@gmail.com
}

Received 27 July 2016; Revised 1 November 2016; Accepted 15 November 2016; Published 12 January 2017

Academic Editor: Patricia J. Y. Wong

Copyright (C) 2017 S. D. Djiomba Njankou and F. Nyabadza. This is an open access article distributed under the Creative Commons Attribution License, which permits unrestricted use, distribution, and reproduction in any medium, provided the original work is properly cited.

\begin{abstract}
A six-compartment mathematical model is formulated to investigate the role of media campaigns in Ebola transmission dynamics. The model includes tweets or messages sent by individuals in different compartments. The media campaigns reproduction number is computed and used to discuss the stability of the disease states. The presence of a backward bifurcation as well as a forward bifurcation is shown together with the existence and local stability of the endemic equilibrium. Results show that messages sent through media have a more significant beneficial effect on the reduction of Ebola cases if they are more effective and spaced out.
\end{abstract}

\section{Introduction}

The world faced one of the most devastating Ebola virus disease (EVD) outbreaks ever in between 2014 and 2015. EVD is caused by a virus called Ebola, which was discovered in the Democratic Republic of Congo in 1976 near a river called Ebola [1]. There are five known species of Ebola: Zaire ebolavirus which has caused the 2014 Ebola disease outbreak [2], Sudan ebolavirus, Cote d'Ivoire ebolavirus, Bundibugyo ebolavirus (Uganda), and Reston ebolavirus which has not yet caused disease in humans [3]. This virus lives in animals like bats and primates, mostly found in Western and Central Africa. The virus can be transmitted from animals to humans when an individual comes into contact with an infectious animal through handling of contaminated meat, for example, and contamination is also possible among animals. Contamination can occur among humans when they have nonprotected contact with an infectious individual's fluids like faeces, vomit, saliva, sweat, and blood [4]. It can also happen in hospitals, where healthcare practitioners paid a heavy price [1].

Symptoms can appear after 2 to 21 days following contamination and the infectious period can last from 4 to 10 days [5]. Some contaminated individuals become symptomatic after 21 days [6], whereas others will never develop symptoms and remain asymptomatic $[4,7,8]$. When the virus gets into a human body, it rapidly replicates and attacks the immune system. So, depending on the state of the infected individual's immune system, death can directly follow or recovery can occur after treatment. According to the World Health Organisation (WHO), a suspected case of EVD is any person, alive or dead, suffering or having suffered from a sudden onset of high fever and having had contact with a suspected or confirmed Ebola case, a dead or sick animal, and at least three of the following symptoms: headaches, anorexia, lethargy, aching muscles or joints, breathing difficulties, vomiting, diarrhoea, stomach pain, inexplicable bleeding, or any sudden inexplicable death [9]. Confirmed cases of EVD are individuals who would have tested positive for the virus antigen either by detection of virus RNA by Reverse Transcriptase Polymerase Chain Reaction or by detection of IgM antibodies directed against Ebola [9]. Ebola seropositive individuals can be either asymptomatic or symptomatic. Post-Ebola survey results showed that $71 \%$ of seropositive individuals monitored were asymptomatic [7]. Symptomless EVD patients have low infectivity due to their very low viral load whereas the symptomatic cases transmit the disease through their fluids [8].

Media campaigns have been included in mathematical models in recent years. Exponential functions are mostly used to represent their impact on people's behaviour which affects disease evolution $[10,11]$.

A model where media coverage influences the transmission rate of a given disease is presented in [12]. An 
exponentially decreasing function is used to describe the media coverage over time. The results show that media coverage has a short-term beneficial effect on the targeted population. A smoking cessation model with media campaign was given in [13] and results showed that the reproduction number is suppressed when media campaigns that focus on smoking cessation are increased. Thus, spreading information to encourage smokers to quit smoking was an effective intervention. The impact of Twitter on influenza was studied in [14]. An exponential term was associated to model the effect of Twitter messages on reducing the transmission rate of influenza. It was noted that Twitter can have a substantial influence on the dynamics of influenza virus infection and can provide a good real-time assessment of the current disease condition.

There is no large-scale treatment for EVD as yet, so stopping the transmission chain remains the only viable form of control. Media campaigns publicise the means of contracting the disease and the behaviour to adopt when a suspected or confirmed Ebola case is detected. The potential effect of media campaigns on Ebola transmission dynamics is thus of great interest. This paper is motivated by the work in [14] and was done as an M.S. research work by the first author [15]. We use a mathematical model to describe the transmission dynamics of EVD in the presence of asymptomatic cases and the impact of media campaigns on the disease transmission is represented by a linear decreasing function. The efficacy of media campaigns is a state variable in this model and a differential equation describing its variation is given. We examine the long-term dynamics of EVD and evaluate the potential impact of media campaigns on reducing the number of Ebola cases. The paper is arranged as follows: the model formulation is presented in Section 2, and the model properties and analysis are given in Section 3. The numerical simulations are presented in Section 4 and we give concluding remarks in the last section.

\section{Model Formulation}

A deterministic model with six independent compartments comprising individuals that are susceptible $(S)$, exposed $(E)$, infected asymptomatic $\left(I_{a}\right)$, infected symptomatic $\left(I_{s}\right)$, recovered $(R)$, and deceased $(D)$ is formulated. The total population size $N$ is given by

$$
\begin{aligned}
& N(t)=S(t)+E(t)+I_{a}(t)+I_{s}(t)+R(t)+D(t) \\
& \forall t \geq 0 .
\end{aligned}
$$

We only consider the Zaire Ebola virus strain which caused the 2014 Ebola outbreak in West Africa. We assume a constant natural death rate $\mu$ for the whole model. The study is made over a relatively large period so that those who recover from EVD gain permanent immunity against the strain.

Recruitment into the susceptibles class is done through birth or migration at a constant rate $\Lambda$ and susceptible individuals become exposed after unsafe contact with Ebola virus. After contamination, susceptibles move to compartment $E$ and, considering $1 / \gamma$ as the incubation period, individuals leave the exposed compartment at a rate $\gamma$. After

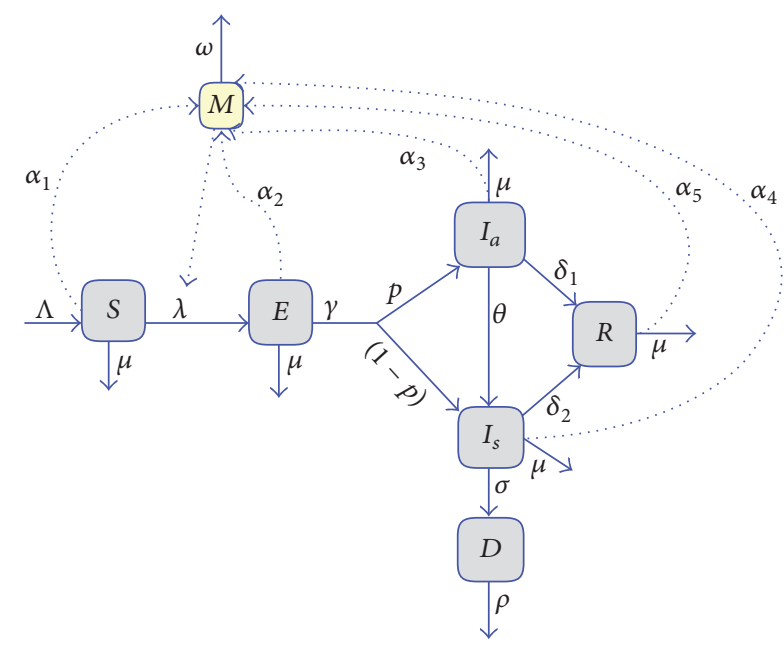

FIGURE 1: Flow diagram for EVD.

the incubation period, a proportion $p$ of the exposed do not develop symptoms and become infected asymptomatic individuals who may recover at a rate $\delta_{1}$. The asymptomatic individuals may develop symptoms and become symptomatic at a rate $\theta$. The rest of the exposed individuals develop symptoms and become symptomatic. The infected symptomatic class is diminished by EVD related deaths at a rate $\sigma$ or recovery at a rate $\delta_{2}$. Recovered individuals can only leave the compartment through natural death and dead bodies are disposed of at a rate $\rho$.

The general objective of media campaigns against a disease is to increase the population's awareness of the disease and correct misperceptions about how it is spread and how it is and is not acquired [18]. The efficacy of messages sent through media is thus their ability to produce the intended results. We consider here that Ebola disease related messages are exchanged by individuals from each of the compartments at any time $t$. After receiving tweets or messages related to Ebola disease, the population decides on the means of preventing or even treating the disease. Messages are assumed to get outdated at a rate $\omega . M(t)$ is defined as the fraction of effective messages sent by individuals of the respective classes at any time $t$. Thus, $M(t)$ is the ratio of effective messages to the total messages sent. The contributions to $M$ from the living compartments are, respectively, $\alpha_{1}, \alpha_{2}, \alpha_{3}, \alpha_{4}$, and $\alpha_{5}$. The use of the campaigns is to reduce EVD transmission. We assume here that media campaigns primarily target the transmission process and $0<M(t) \leq 1, \forall t \geq 0$.

The force of infection will be given by

$$
\lambda(t)=\beta c(1-M(t)) \frac{\left(I_{s}(t)+\eta D(t)\right)}{N(t)},
$$

where $\beta$ is the probability that a contact will result in an infection and $c$ is the number of contacts between susceptible and infectious individuals. The parameter $\eta>1$ describes the high infectivity of dead bodies. The flow diagram is presented in Figure 1. 
2.1. Model Equations. The system of differential equations describing the variation of the state variables within the model is as follows:

$$
\begin{aligned}
& \frac{d S(t)}{d t}=\Lambda-(\mu+\lambda(t)) S(t) \\
& \frac{d E(t)}{d t}=\lambda(t) S(t)-(\mu+\gamma) E(t) \\
& \frac{d I_{a}(t)}{d t}=p \gamma E(t)-\left(\mu+\theta+\delta_{1}\right) I_{a}(t) \\
& \frac{d I_{s}(t)}{d t}=(1-p) \gamma E(t)+\theta I_{a}(t)-\left(\mu+\delta_{2}+\sigma\right) I_{s}(t) \\
& \frac{d R(t)}{d t}=\delta_{1} I_{a}(t)+\delta_{2} I_{s}(t)-\mu R(t) \\
& \frac{d D(t)}{d t}=\sigma_{s}(t)-\rho D(t) \\
& \frac{d M(t)}{d t}=\alpha_{1} S(t)+\alpha_{2} E(t)+\alpha_{3} I_{a}(t)+\alpha_{4} I_{s}(t) \\
& \quad+\alpha_{5} R(t)-\omega M(t) .
\end{aligned}
$$

We set $S(0)>0, E(0) \geq 0, I_{a}(0) \geq 0, I_{s}(0) \geq 0, R(0) \geq$ $0, D(0) \geq 0$, and $M(0) \geq 0$ as the initial values of each of the state variables $S, E, I_{a}, I_{s}, R, D$, and $M$, all assumed to be positive.

\section{Model Properties and Analysis}

3.1. Existence and Uniqueness of Solutions. The right hand side of system (3)-(9) is made of Lipschitz continuous functions since they describe the size of a population. According to Picard's Existence Theorem, with given initial conditions, the solutions of our system exist and they are unique.

Theorem 1. The system makes biological sense in the region

$$
\begin{aligned}
\Omega & =\left\{\left(S(t), E(t), I_{a}(t), I_{s}(t), R(t), D(t), M(t)\right)\right. \\
& \left.\in R^{7}: N(t) \leq \frac{\Lambda}{\mu}, 0<M(t) \leq 1\right\}
\end{aligned}
$$

which is attracting and positively invariant with respect to the flow of system (3)-(9).

Proof. We first assume that $\rho>\mu$ during the modelling time. This assumption makes sense since EVD death rate is higher than the natural death rate in the course of an EVD epidemic. By adding (3)-(8), we have

$$
\frac{d N(t)}{d t} \leq \Lambda-\mu N(t)
$$

Integrating (11) gives the following solution:

$$
0 \leq N(t) \leq\left(N(0)-\frac{\Lambda}{\mu}\right) \exp [-\mu t]+\frac{\Lambda}{\mu}, \quad \forall t \geq 0 .
$$

We have $\lim _{t \rightarrow \infty} N(t)<\Lambda / \mu$ when $N(0) \leq \Lambda / \mu$. However, if $N(0) \geq \Lambda / \mu, N(t)$ will decrease to $\Lambda / \mu$. So, $N(t)$ is thus a bounded function of time.

Together with $M$ which is already bounded (see proof in Appendix A), we can say that $\Omega$ is bounded and at limiting equilibrium $\lim _{t \rightarrow \infty} N(t)=\Lambda / \mu$. Besides, any sum or difference of variables in $\Omega$ with positive initial values will remain in $\Omega$ or in a neighbourhood of $\Omega$. Thus, $\Omega$ is positively invariant and attracting with respect to the flow of system (3)(9).

\subsection{Positivity of Solutions}

Theorem 2. The existing solutions of system (3)-(9) are all positive.

Proof. From (3), we can have

$$
\frac{d S(t)}{d t} \geq-(\lambda(t)+\mu) S(t), \quad \forall t \geq 0 .
$$

Solving for (13) yields

$$
S(t)=S(0) \exp \left[-\int_{0}^{t} \lambda(\tau) d \tau-\mu t\right]
$$

which is positive given that $S(0)$ is also positive.

Similarly, from (4), we have

$$
\frac{d E(t)}{d t} \geq-(\gamma+\mu) E(t), \quad \forall t \geq 0
$$

so that

$$
E(t)=E(0) \exp [-(\gamma+\mu) t]
$$

which thus shows that $E(t)$ is positive since $E(0)$ is also positive.

Similarly, from (5), we can write

$$
\frac{d I_{a}(t)}{d t} \geq-\left(\mu+\theta+\delta_{1}\right) I_{a}(t), \quad \forall t \geq 0,
$$

from which we obtain

$$
I_{a}(t) \geq I_{a}(0) \exp \left[-\left(\mu+\theta+\delta_{1}\right) t\right]
$$

Thus, $I_{a}$ is positive since $I_{a}(0)$ is positive.

The remaining equations yield

$$
\begin{aligned}
I_{s}(t) & \geq I_{s}(0) \exp \left[-\left(\mu+\sigma+\delta_{2}\right) t\right], \\
R(t) & \geq R(0) \exp [-\mu t], \\
D(t) & \geq D(0) \exp [-\rho t], \\
M(t) & \geq M(0) \exp [-\omega t] .
\end{aligned}
$$

So, $I_{s}(t), R(t)$, and $M(t)$ are all positive for positive initial conditions. Thus, all the state variables are positive. 
3.3. Steady States Analysis. This model has two steady states: the disease-free equilibrium (DFE) which describes the total absence of EVD in the studied population and the endemic equilibrium (EE) which exists at any positive prevalence of EVD in the population. This section is dedicated to the study of local and global stability of these steady states.

3.4. The Disease-Free Equilibrium and $R_{M}$. The diseasefree equilibrium is given by $\left(S^{*}, E^{*}, I_{a}^{*}, I_{s}^{*}, R^{*}, D^{*}, M^{*}\right)=$ $\left(\Lambda / \mu, 0,0,0,0,0, \Lambda \alpha_{1} / \omega \mu\right)$. To compute the media campaigns reproduction number $R_{M}$, we use the next generation method comprehensively discussed in [19]. The renewal matrix $F$ and transfer matrix $V$ at DFE are

$$
\begin{aligned}
& F=\left[\begin{array}{cccc}
0 & 0 & c \beta\left(1-M^{*}\right) & c \beta \eta\left(1-M^{*}\right) \\
0 & 0 & 0 & 0 \\
0 & 0 & 0 & 0 \\
0 & 0 & 0 & 0
\end{array}\right], \\
& V=\left[\begin{array}{cccc}
Q_{1} & 0 & 0 & 0 \\
-\gamma p & Q_{2} & 0 & 0 \\
(p-1) \gamma & -\theta & Q_{3} & 0 \\
0 & 0 & \sigma & -\rho
\end{array}\right]
\end{aligned}
$$

where

$$
\begin{aligned}
& Q_{1}=\gamma+\mu, \\
& Q_{2}=\mu+\theta+\delta_{1}, \\
& Q_{3}=\delta_{2}+\sigma+\mu .
\end{aligned}
$$

The media campaigns reproduction number $R_{M}$ is the spectral radius of the matrix $F V^{-1}$ and is given by

$$
R_{M}=\frac{c \beta \gamma\left(1-M^{*}\right)}{\rho Q_{1} Q_{2} Q_{3}}\left(p \theta+(1-p) Q_{2}\right)(\rho+\eta \sigma) .
$$

We can rewrite $R_{M}=R_{1}+R_{2}$ for elucidation purposes where

$$
\begin{aligned}
& R_{1}=\frac{c \beta \gamma\left(1-M^{*}\right)}{\rho Q_{1} Q_{3}}(1-p \nu), \\
& R_{2}=\frac{c \beta \gamma\left(1-M^{*}\right)}{\rho Q_{1} Q_{3}}(1-p \nu) \eta \sigma,
\end{aligned}
$$

and $\nu=\left(\mu+\delta_{1}\right) / Q_{2}$.

Note here that $\gamma / Q_{1}$ is the probability that an individual in $E$ moves either to $I_{a}$ or to $I_{s} \cdot \sigma / Q_{3}$ is the proportion of symptomatic individuals who die from EVD. Thus, the media campaigns reproduction number is a sum of secondary infections due to infectious individuals in $I_{s}$ and the deceased in $D$. Notice here the reduction factor $1-M^{*}$ which represents the attenuating effect of media campaigns on the future number of EVD cases.

Theorem 3. The DFE is globally asymptotically stable whenever $R_{M}<R_{M}^{c}<1$, where $R_{M}^{c}=\min (R(M(t)), R(M, v))$ and $R(M, v)$ will be defined later. When $R_{M}^{c}<R_{M}<1$, the DFE is locally stable. Otherwise, the DFE is unstable.
Proof. Let us define $V(t)=E(t)+I_{a}(t)+I_{s}(t)+D(t)$ as the Lyapunov function.

$$
\begin{aligned}
& V(t)>0 \text { since } E(t)>0, I_{a}(t)>0, I_{s}(t)>0, \text { and } \\
& D(t)>0 \forall t>0 . \\
& V(t)=0 \text { if } E(t)=I_{a}(t)=I_{s}(t)=D(t)=0(\text { at DFE }) .
\end{aligned}
$$

Thus, $V$ is a positive definite function at the DFE.

The derivative of $V$ is given by

$$
\begin{aligned}
\dot{V}= & \dot{E}+\dot{I}_{a}+\dot{I}_{s} \\
= & \left(c \beta(1-M) \frac{S}{N}-Q_{3}+\sigma\right) I_{s}+\left(\gamma-Q_{1}\right) E \\
& +\left(\theta-Q_{2}\right) I_{a}-\rho D .
\end{aligned}
$$

Also, $S / N \leq 1$ and at equilibrium

$$
\begin{aligned}
E & =\frac{Q_{2}}{p \gamma} I_{a}, \\
I_{s} & =\frac{\left[p \theta+(1-p) Q_{2}\right]}{p Q_{3}} I_{a}, \\
D & =\frac{\sigma}{\rho} I_{s} .
\end{aligned}
$$

Plugging (25) into (24) yields

$$
\dot{V} \leq \frac{Q_{1} Q_{2} Q_{3}}{\gamma\left[p \theta+(1-p) Q_{2}\right]}(R(M(t))-1) I_{s}
$$

with

$$
\begin{aligned}
& R(M(t)) \\
& \quad=\frac{c \beta \gamma(1-M(t))}{\rho Q_{1} Q_{2} Q_{3}}\left(p \theta+(1-p) Q_{2}\right)(\rho+\eta \sigma) .
\end{aligned}
$$

Thus, $\dot{V} \leq 0$ when $R(M(t)) \leq 1$ and, particularly, $\dot{V}=0$ only if $E=I_{a}=I_{s}=D=0$. Since $M(t) \geq M^{*}$ for all $t>0$ (see proof in Appendix A), we have $R_{M}<R(M(t))$. Because the largest invariant set for which $\dot{V}=0$ in $\Omega$ is the DFE and $\dot{V} \leq 0$ if $R(M(t)) \leq 1$, by using the invariance principle of LaSalle [20], we can conclude that the DFE is globally asymptotically stable for $R_{M}<R(M(t))<1$. Together with the existence of a backward bifurcation later proven, we finally obtain the global stability of the DFE for $R_{M}<R_{M}^{c}<1$.

Analysis of the Reproduction Number $R_{M} \cdot R_{M}$ is considered as a reproduction number whose values depend on the fraction of effective messages on EVD at a given time. Assuming $M$ to be constant, Figure 2 graphically describes the relationship between two concepts: reproduction number and media campaigns efficacy. It shows the reducing effect of media campaigns on the number of EVD infected individuals and indicates as well how we can test the efficacy of Ebola related messages through the pace of the disease transmission. In fact, for each value of $M$, the corresponding value of 


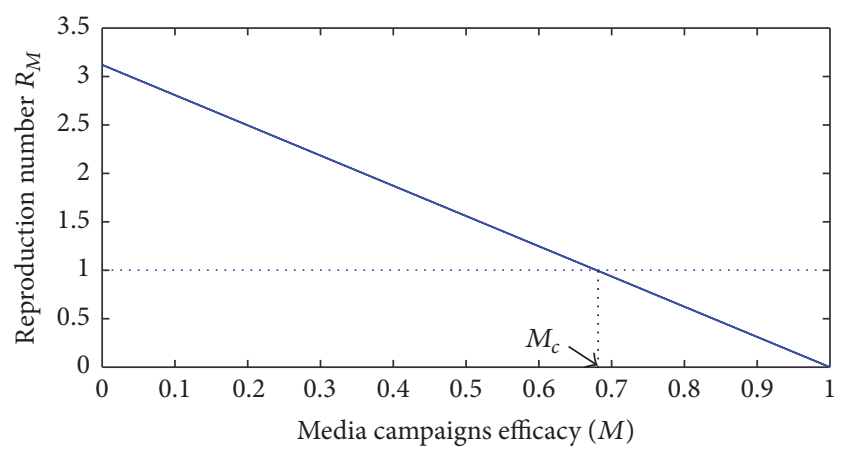

Figure 2: Time dependent reproduction number. The parameters values used for this plot are $\mu=0.008, \beta=0.2, \sigma=0.58, \gamma=0.845$, $p=0.85, \theta=0.1, \delta_{1}=0.15, \delta_{2}=0.6, c=12, \omega=4 \times 10^{-4}$, $\alpha_{1}=9 \times 10^{-7}, \alpha_{2}=2 \times 10^{-7}, \alpha_{3}=5 \times 10^{-6}, \alpha_{4}=8 \times 10^{-5}, \alpha_{5}=10^{-6}$, $\rho=0.97$, and $\eta=3.5$.

the reproduction number can be found and then used to analyse the disease evolution. For instance, when $R_{M}=1$, the critical value of media campaigns efficacy $M_{c}$ can be determined. Since the behaviour of the system changes when the reproduction number crosses the value one, $M_{c}$ can also be used as a threshold parameter that indicates a behavioural change of the system and thus can help in the disease control for any given set of parameter values.

3.5. Existence and Stability of the Endemic Equilibrium. In this section, we show the existence of the endemic equilibrium (EE). We denote the endemic equilibrium by $\left(S^{* *}, E^{* *}, I_{a}^{* *}, I_{s}^{* *}, R^{* *}, D^{* *}, M^{* *}\right)$. At equilibrium, (3)-(9) give

$$
\begin{aligned}
S^{* *} & =\frac{1}{\lambda^{* *}+\mu} Q_{1} Q_{2} Q_{3}, \\
E^{* *} & =\frac{\lambda^{* *}}{\left(\lambda^{* *}+\mu\right)} Q_{2} Q_{3}, \\
I_{a}^{* *} & =\frac{p \gamma \lambda^{* *}}{\left(\lambda^{* *}+\mu\right)} Q_{3}, \\
I_{s}^{* *} & =\frac{\gamma \lambda^{* *}\left[p \theta+Q_{2}(1-p)\right]}{\left(\lambda^{* *}+\mu\right)}, \\
R^{* *} & =\frac{\gamma \lambda^{* *}\left[p\left(Q_{3} \delta_{1}+\theta \delta_{2}\right)+Q_{2} \delta_{2}(1-p)\right]}{\mu\left(\lambda^{* *}+\mu\right)} \\
D^{* *} & =\frac{\gamma \lambda^{* *} \sigma\left[p \theta+Q_{2}(1-p)\right]}{\rho\left(\lambda^{* *}+\mu\right)}, \\
M^{* *} & =\frac{1}{\mu \omega\left(\lambda^{* *}+\mu\right)}\left(\phi_{1}+\phi_{2} \lambda^{* *}\right),
\end{aligned}
$$

where

$$
\begin{aligned}
\lambda^{* *} & =\beta c\left(1-M^{* *}\right) \frac{\left(I_{s}^{* *}+\eta D^{* *}\right)}{N^{* *}}, \\
\phi_{1} & =\mu Q_{1} Q_{2} Q_{3} \alpha_{1},
\end{aligned}
$$

TABLE 1: Roots signs.

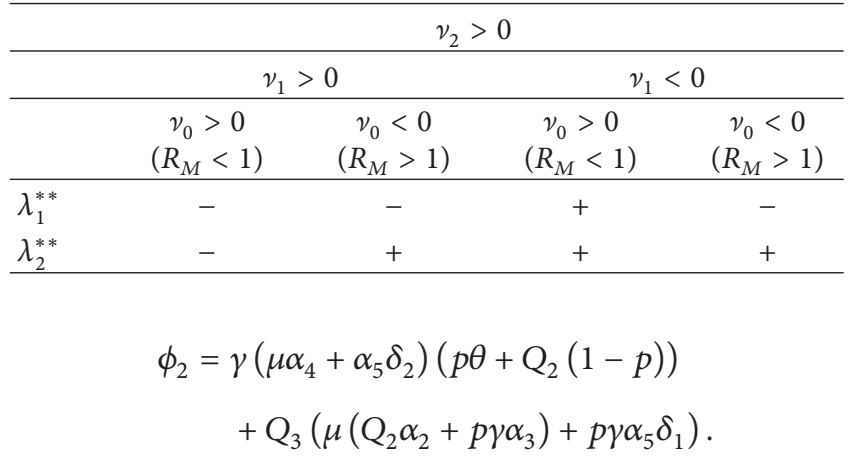

Set $P\left(\lambda^{* *}\right)=\lambda^{* *}-\beta c\left(1-M^{* *}\right)\left(\left(I_{s}^{* *}+\eta D^{* *}\right) / N^{* *}\right)$. By replacing $M^{* *}, I_{s}^{* *}, D^{* *}$, and $N^{* *}$ by their values expressed as functions of $\lambda^{s *}$ and by setting

$$
P\left(\lambda^{* *}\right)=0
$$

we obtain the following equation:

$$
\lambda^{* *}\left[\left(v_{2}\left(\lambda^{* *}\right)^{2}+v_{1} \lambda^{* *}+v_{0}\right)\right]=0,
$$

where

$$
\begin{aligned}
v_{0} & =\mu^{2} \omega \rho Q_{1}^{2} Q_{2}^{2} Q_{3}^{2}\left(1-R_{M}\right), \\
v_{1} & =Q_{1} Q_{2} Q_{3}\left(\xi_{1}+\xi_{2}\right) \mu \omega+\xi_{3}, \\
v_{2} & =Q_{1} Q_{2} Q_{3}\left[\gamma\left(\mu(\rho+\sigma)+\rho \delta_{2}\right)\left(p \theta+(1-p) Q_{2}\right)\right. \\
& \left.+\rho Q_{3}\left(\mu Q_{2}+p \gamma\left(\mu+\delta_{1}\right)\right)\right] \omega,
\end{aligned}
$$

with

$$
\begin{aligned}
\xi_{1} & =\rho\left(1+Q_{1} Q_{2} Q_{3}+Q_{2} Q_{3} \mu+p \gamma Q_{3} \mu\right), \\
\xi_{2} & =\left(p \theta+(1-p) Q_{2}\right)(\rho(-\beta c \gamma+\gamma \mu) \\
& +\gamma \sigma(-\beta c \eta+\mu)), \\
\xi_{3} & =\beta c \gamma \Lambda \mu\left(p \theta+(1-p) Q_{2}\right)(\rho+\eta \sigma) \\
& \cdot\left(Q_{3}\left(Q_{2} \alpha_{2}+p \gamma \alpha_{3}\right)+\left(p \theta+(1-p) Q_{2}\right) \alpha_{4}\right. \\
& \left.-\left(p Q_{3} \delta_{1}+\left(p \theta+(1-p) Q_{2}\right) \frac{\delta_{2}}{\mu}\right) \alpha_{5}\right) .
\end{aligned}
$$

From (31), $\lambda^{* *}=0$ corresponds to the DFE discussed in the previous section. The signs of the solutions of the quadratic equation

$$
v_{2}\left(\lambda^{* *}\right)^{2}+\nu_{1} \lambda^{* *}+v_{0}=0
$$

are given in Table 1.

From Table 1, we notice that, for the existence and uniqueness of the endemic equilibrium, $v_{0}$ must be negative. This is only possible if $R_{M}>1$. Thus, we have the following theorem on the existence of the endemic equilibrium. 
Theorem 4. (i) If $R_{M}>1$, (34) has a unique positive solution and system (3)-(9) has a unique endemic equilibrium.

(ii) If $R_{M}^{c}<R_{M}<1$ and $\nu_{1}<0$, the roots $\lambda_{1}^{* *}$ and $\lambda_{2}^{* *}$ are both positive, and system (3)-(9) admits two endemic equilibria.

(iii) If $R_{M}^{c}=R_{M}$, then (34) has a repeated positive root and a unique endemic equilibrium exists for system (3)-(9).

(iv) If $0<R_{M}<R_{M}^{c}$, then system (3)-(9) does not admit any endemic equilibrium and only the DFE exists.

Provided $v_{1}<0$, the existence of two endemic equilibria for $R_{M}<1$ suggests the existence of a backward bifurcation since the DFE also exists in that particular domain. The coexistence of DFE and endemic equilibrium when $R_{M}<$ 1 is a well known characteristic of a backward bifurcation

$0<R_{M}<R_{M}^{c}$, the DFE is globally stable,

$R_{M}^{c}<R_{M}<1$

the DFE is locally stable and two endemic equilibria exist with one which is stable and the other one unstable.

The DFE and EE both describe different qualitative behaviours of our epidemic. Let us set $\phi=c \beta\left(1-M^{*}\right)$ as our bifurcation parameter, so that

$$
\phi=\phi^{*}=\frac{\rho Q_{1} Q_{2} Q_{3}}{\gamma\left(p \theta+(1-p) Q_{2}\right)(\rho+\eta \sigma)},
$$

$$
\text { for } R_{M}=1 \text {. }
$$

In order to describe the stability of the endemic equilibrium, we use the theorem, remark, and corollary in [22] which are based on the Centre Manifold Theory, and formulated in Appendix B.

Theorem 5. A unique endemic equilibrium exists when $R_{M}>$ 1 and is locally asymptotically stable.

Proof. For model (3)-(9), the DFE $\left(E_{0}\right)$ is not equal to zero. According to Remark 1 in [22], we notice that if the equilibrium of interest in Theorem B.1 is a nonnegative equilibrium $x_{0}$, then the requirement that $w$ is nonnegative in Theorem B.1 is not necessary. When some components in $w$ are negative, one can still apply Theorem B.1 on condition that

$$
w(j)>0, \quad \text { if } x_{0}(j)=0,
$$

if $x_{0}(j)>0, w(j)$ does not need to be positive,

where $w(j)$ and $x_{0}(j)$ denote the $j$ th component of $w$ and $x_{0}$, respectively.

Firstly, let us rewrite system (3)-(9) introducing

$$
\begin{gathered}
S=x_{1}, \\
E=x_{2}, \\
I_{a}=x_{3},
\end{gathered}
$$

described in [21]. Thus, there exists a critical value of $R_{M}$, denoted by $R_{M}^{c}$, for which there is a change in the qualitative behaviour of our model.

At the bifurcation point, there is an intersection between the line $R_{M}=R_{M}^{c}$ and the graph of $P\left(\lambda^{* *}\right)$. The discriminant $\Delta$ is equal to zero at $R_{M}=R(M, v)$, which is solution of

$$
v_{1}^{2}-4 \omega Q_{1} Q_{2} Q_{3} \mu(1-R(M, \nu)) \nu_{2}=0 .
$$

Equation (35) implies

$$
R(M, v)=1-\frac{v_{1}^{2}}{4 \psi \nu_{2}} .
$$

Considering as well the threshold value of the reproduction number from Theorem 3, we can conclude that $R_{M}^{c}=$ $\min (R(M(t)), R(M, v))$. So, 
The eigenvalues of $A$ are $-\mu$ (twice), $-\omega, 0$, and the roots of polynomial (42) below:

$$
Q(\varsigma)=\varsigma^{3}+d_{0} \varsigma^{2}+d_{1} \varsigma+d_{2}
$$

where

$$
\begin{aligned}
d_{0}= & \rho+Q_{1}+Q_{2}+Q_{3}, \\
d_{1}= & Q_{1}\left(Q_{2}+Q_{3}\right)+Q_{2} Q_{3}+\rho\left(Q_{1}+Q_{2}+Q_{3}\right) \\
& -\phi^{*}(1-p) \gamma, \\
d_{2}= & Q_{1} Q_{2} Q_{3}+Q_{2} Q_{3} \rho+Q_{1}\left(Q_{2}+Q_{3}\right) \rho \\
& -\gamma\left(p \theta+(1-p)\left(Q_{2}+\rho+\eta \sigma\right)\right) \phi^{*} .
\end{aligned}
$$

Our linearisation matrix $A$ will thus have zero as simple eigenvalue. Statement (A1) is verified. We now show that (A2) is satisfied.

The right eigenvector $W=\left[w_{1}, w_{2}, w_{3}, w_{4}, w_{5}\right]^{\prime}$ and the left eigenvector $V=\left[v_{1}, v_{2}, v_{3}, v_{4}, v_{5}, v_{6}\right]$ associated with the eigenvalue 0 such that $V W=1$ are solutions of the system:

$$
\begin{aligned}
& A W=[0,0,0,0,0,0]^{\prime}, \\
& V A=[0,0,0,0,0,0]^{\prime}, \\
& V W=1 . \\
& \psi_{1}=\left(p \theta+(1-p) Q_{2}\right) \gamma \sigma, \\
& \psi_{2}=-\rho(\rho+\eta \sigma)\left(\frac{Q_{2} Q_{3} \psi_{1}}{\gamma \sigma}+Q_{1} p Q_{3} \theta\right)+\left(\rho^{2}\right. \\
& \left.+\eta \sigma\left(Q_{3}+\rho\right)\right)\left(Q_{1}(p-1) Q_{2}^{2}-p Q_{2} \theta\right), \\
& \psi_{3}=2 Q_{1} Q_{2} Q_{3} \rho(\rho+\eta \sigma) \omega\left(\mu \left(Q_{1} Q_{2}+(\rho+\sigma)(p \theta\right.\right. \\
& \left.\left.+(1-p) Q_{2}\right)+p \gamma Q_{3} \rho\right) \omega+\rho\left(\mu Q_{2} Q_{3} \alpha_{2}\right. \\
& +\gamma\left(p Q_{3} \mu \alpha_{3}+\left(p \theta+(1-p) Q_{2}\right) \mu \alpha_{4}\right. \\
& \left.\left.\left.+\left(\omega+\alpha_{5}\right)\left(p Q_{3} \delta_{1}+\left(p \theta+(1-p) Q_{2}\right) \delta_{2}\right)\right)\right)\right), \\
& \psi_{4}=\gamma \Lambda \sigma\left(-p Q_{3} \rho^{2} \theta-Q_{2}\left(p \theta+(1-p) Q_{2}\right) \rho^{2}\right. \\
& +Q_{1} \eta\left(-p Q_{3} \theta \rho-Q_{2}\left(Q_{3}+\rho\right)\left(p \theta+(1-p) Q_{2}\right)\right) \\
& \left.\cdot \sigma-Q_{2} Q_{3}\left(p \theta+(1-p) Q_{2}\right) \rho(\rho+\eta \sigma)\right)(\omega \\
& \left.+\alpha_{1}\right)^{2} \text {, } \\
& \psi_{5}=Q_{2} Q_{3} \rho\left(-Q_{1} \alpha_{1}+\mu \alpha_{2}\right)+\gamma \rho\left(p Q_{3}\left(\mu \alpha_{3}+\delta_{1} \alpha_{5}\right)\right. \\
& \left.+\left(p \theta+(1-p) Q_{2}\right) \gamma \rho\left(\mu \alpha_{4}+\delta_{2} \alpha_{5}\right)\right),
\end{aligned}
$$

we have

$$
\begin{aligned}
& w_{1}=\frac{-Q_{1} Q_{2} Q_{3} \rho}{\mu \psi_{1}}, \\
& v_{1}=0 \\
& w_{2}=\frac{\rho Q_{2} Q_{3}}{\psi_{1}}, \\
& v_{2}=\frac{-\psi_{1}\left(p \theta+(1-p) Q_{2}\right)(\rho+\eta \sigma)}{\psi_{2}} \\
& w_{3}=\frac{\rho \gamma p Q_{3}}{\psi_{1}}, \\
& v_{3}=\frac{-\theta \psi_{1}(\rho+\eta \sigma) Q_{1}}{\gamma \psi_{2}}, \\
& w_{4}=\frac{\rho}{\sigma}, \\
& w_{7}=\frac{-\eta Q_{1} Q_{2} Q_{3} \psi_{1}}{\omega \psi_{1} \mu}, \\
& v_{7}=0 \\
& v_{6}=\frac{-Q_{1} Q_{2}(\rho+\eta \sigma)}{\gamma \psi_{2}}, \\
& w_{5}=\frac{1}{\mu}\left(\frac{p \gamma Q_{3} \rho \delta_{1}}{\psi_{1}}-\frac{\rho \delta_{2}}{\sigma}\right) \\
& v_{5} \\
& =0
\end{aligned}
$$

We notice that

$$
\begin{aligned}
& E_{0}\left(x_{2}\right)=0, \quad w_{2}>0, \\
& E_{0}\left(x_{3}\right)=0, \quad w_{3}>0, \\
& E_{0}\left(x_{4}\right)=0, \quad w_{4}>0, \\
& E_{0}\left(x_{5}\right)=0, \quad w_{5}>0, \\
& E_{0}\left(x_{6}\right)=0, \quad w_{6}>0 .
\end{aligned}
$$

Besides, since $E_{0}\left(x_{1}\right)$ and $E_{0}\left(x_{7}\right)$ are positive, $w_{1}$ and $w_{7}$ do not need to be positive according to Remark 1 in [22]. So, statement (A2) is verified.

The formulas of the constants $a$ and $b$ are

$$
\begin{aligned}
& a=\sum_{k, i, j=1}^{n} v_{k} w_{i} w_{j} \frac{\partial^{2} f_{k}}{\partial x_{i} \partial x_{j}}\left(E_{0}, \phi^{*}\right), \\
& b=\sum_{k, i=1}^{n} v_{k} w_{i} \frac{\partial^{2} f_{k}}{\partial x_{i} \partial \phi}\left(E_{0}, \phi^{*}\right) .
\end{aligned}
$$




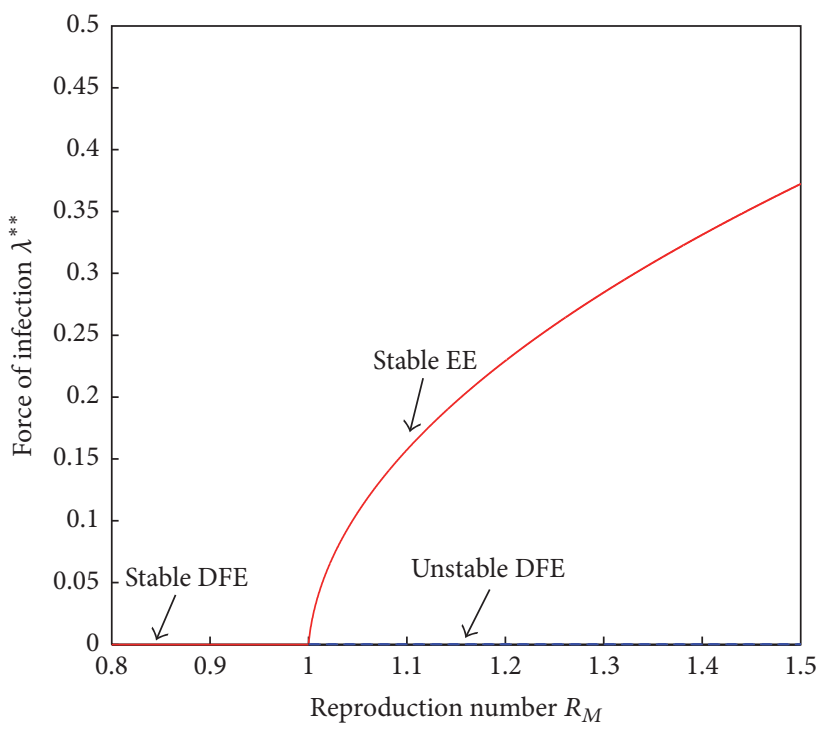

(a)

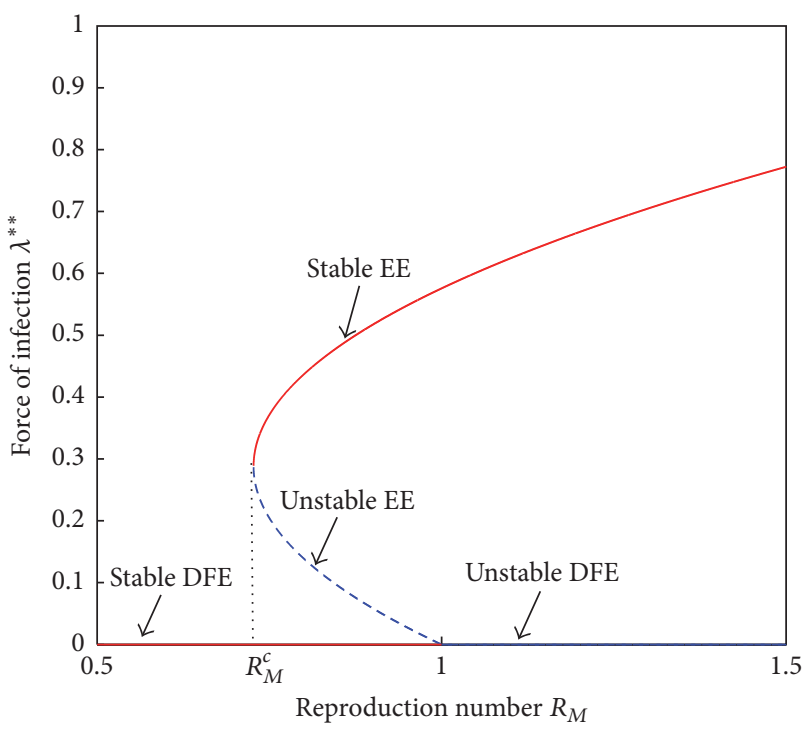

(b)

FIgURE 3: Forward bifurcation for $R_{M}=3.11$ in (a) and backward bifurcation for $R_{M}=0.95$ in (b) with $R_{M}^{c}=0.68$.

After multiple derivations, we have

$$
\begin{aligned}
& a=\frac{\psi_{3}}{\psi_{4}}<0, \\
& b=\frac{\gamma\left(p-\theta+(1-p) Q_{2}\right)^{2}(\rho+\eta \sigma) \omega}{-\psi_{2}}>0 .
\end{aligned}
$$

Since $a<0$ and $b>0$, by using the fourth item of Theorem B.1, we can conclude that when $\phi^{*}$ changes from negative to positive, $E_{0}$ changes its stability from stable to unstable. Correspondingly, a negative unstable equilibrium becomes positive and locally asymptotically stable and a forward bifurcation appears [21].

3.6. Bifurcation Analysis. The study of the DFE and EE led to the proof of the existence of a backward and a forward bifurcation for our model. Graphically, they are, respectively, represented in Figures 3(a) and 3(b) where $R_{M}$ is chosen as the bifurcation parameter. We have shown that a forward bifurcation exists for values of $R_{M}$ greater than one. This means that EVD will persist as long as secondary infections will occur and reducing $R_{M}$ to values less than one is enough to eradicate EVD. However, the existence of a backward bifurcation makes it difficult to control the epidemic. In fact, the coexistence of the DFE and the EE for $R_{M}$ in $\left[R_{M}^{c}, 1\right]$ shows that reducing the number of secondary infections to less than one is not enough to eradicate EVD. Other control measures like quarantine and contact tracing should be implemented together with media campaigns to reach a globally stable DFE and wipe out EVD.

Figure 4 shows time series plots for the force of infection $\lambda$ for varying initial conditions. The trajectories converge to steady states depending on the initial conditions and the values of $R_{M}$. We can observe that when the DFE is asymptotically stable $\left(R_{M}<R_{M}^{c}\right)$, the force of infection reaches

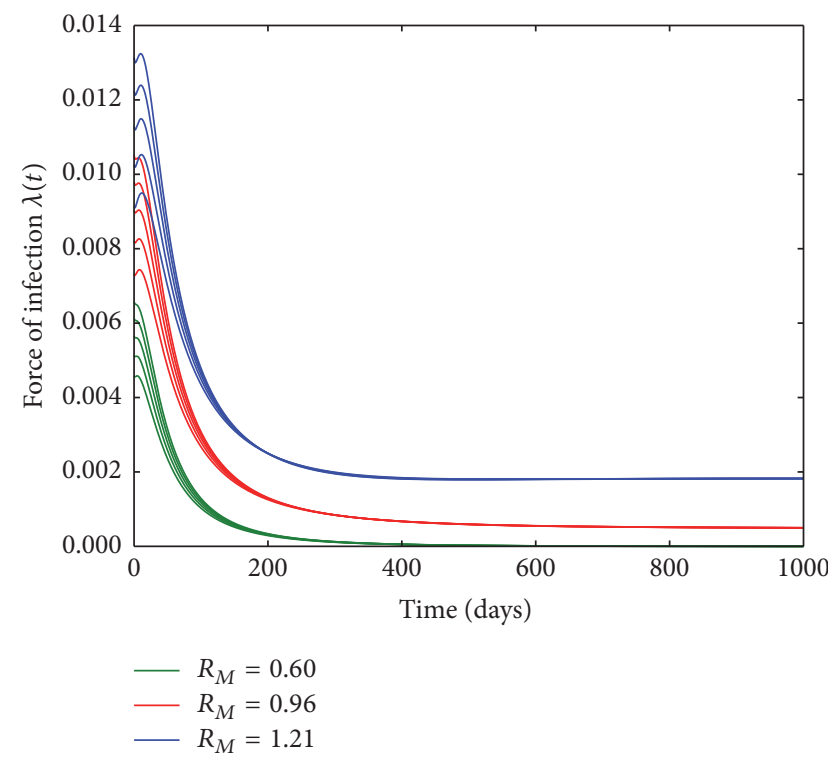

FIGURE 4: Time series variation of the force of infection for different values of the reproduction number.

zero. When the DFE is locally stable and the EE is unstable $\left(R_{M}^{c}<R_{M}<1\right)$, the force of infection reflects a persistent infection. When the EE is unstable $\left(R_{M}>1\right)$, the force of infection is maximal. This confirms the results obtained at the bifurcation analysis and describes the unstable nature of EVD which can easily become an explosive epidemic after a small increase in its force of infection as $R_{M}$ passes through 1 .

\section{Numerical Simulations}

In this section, we use Matlab to carry out simulations for our model. We first verify our theoretic conclusions related 
to stability analysis of system (3)-(9) and then we vary our parameters values to better understand how media campaigns influence the prevalence and transmission of EVD.

It is important to note that the figures chosen are for illustrative purposes only, as we endeavour to verify the analytic results.

4.1. Parameters' Estimation. The parameters used in the simulations are either obtained from the literature or estimated. Since the mean infectious period is set to be from 4 to 10 days, the highest recovery rate $\delta_{2}$ is set to $1 / 4$. The recovery rate of asymptomatic individuals is assumed to be greater than the one of the symptomatic individuals since the former have stronger resistance to EVD. Without any reliable source for EVD media related data, we assume that individuals can send EVD related messages through media independently of their disease status. At the beginning of the epidemic, there is neither a recovered nor an asymptomatic infected individual since only symptomatic persons transmit the disease. We also assume that messages are transmitted through media at time $t=0$, at least for preventive purpose. The setting of the initial conditions is driven by the fact that the population of Nzérékoré, the region where this 2014 Ebola disease outbreak started in Guinea, is estimated to be 1,663,582 individuals [23]. We consider the introduction of infectives in the population and high infectivity of dead bodies $(\eta=1.5)$. The initial conditions are then

$$
\begin{aligned}
& S_{0}=990000, \\
& E_{0}=8000, \\
& I_{a 0}=0, \\
& I_{s 0}=2000, \\
& R_{0}=0, \\
& D_{0}=0, \\
& M_{0}=0.4 .
\end{aligned}
$$

Table 2 gives the description of parameters and their values.

4.2. Sensitivity Analysis. In mathematical modelling, parameters whose values are not precisely known are often used and may vary within some ranges. Numerical methods used to solve equations derived from models may introduce numerical errors in the results. The effects of such errors or uncertainties in the model's parameters are quantified through sensitivity analysis. The aim of sensitivity analysis is to quantify the influence of parameters variation on calculated results [24].

Sensitivity indices allow us to measure the relative change in a state variable when a parameter changes. The normalized

\begin{tabular}{|c|c|c|c|}
\hline Parameters & s Description (per day) & Range & Source \\
\hline$\Lambda$ & Recruitment rate & 20000 & Estimated \\
\hline$\beta$ & $\begin{array}{l}\text { Probability for a contact } \\
\text { to be infectious }\end{array}$ & {$[0.02,1]$} & {$[7]$} \\
\hline$c$ & Number of contacts & {$[1,500]$} & Estimated \\
\hline$\gamma$ & $\begin{array}{c}\text { Rate of exposed } \\
\text { individuals becoming } \\
\text { infectious }\end{array}$ & {$[0.04,0.5]$} & {$[5]$} \\
\hline$\mu$ & Natural death rate & 0.2 & Estimated \\
\hline$p$ & $\begin{array}{c}\text { Proportion of } \\
\text { asymptomatic infected } \\
\text { individuals }\end{array}$ & {$[0.15,0.7]$} & {$[7]$} \\
\hline$\theta$ & $\begin{array}{c}\text { Rate of asymptomatic } \\
\text { individuals becoming } \\
\text { symptomatic }\end{array}$ & 0.12 & {$[6]$} \\
\hline$\sigma$ & Disease related death rate & {$[0.2,0.9]$} & {$[1]$} \\
\hline$\rho$ & $\begin{array}{c}\text { Disposal rate of dead } \\
\text { bodies }\end{array}$ & 0.497 & {$[16]$} \\
\hline$\delta_{1}$ & $\begin{array}{c}\text { Recovery rate of } \\
\text { asymptomatic individuals }\end{array}$ & {$[0,0.6]$} & Estimated \\
\hline$\delta_{2}$ & $\begin{array}{c}\text { Recovery rate of } \\
\text { symptomatic individuals }\end{array}$ & {$[0,0.25]$} & [5] \\
\hline$\alpha_{1}$ & $\begin{array}{l}\text { Rate of messaging by } \\
\text { susceptible individuals }\end{array}$ & {$\left[0,10^{-1}\right]$} & Estimated \\
\hline$\alpha_{2}$ & $\begin{array}{l}\text { Rate of messaging by } \\
\text { exposed individuals }\end{array}$ & {$\left[0,10^{-1}\right]$} & Estimated \\
\hline$\alpha_{3}$ & $\begin{array}{l}\text { Rate of messaging by } \\
\text { infected asymptomatic } \\
\text { individuals }\end{array}$ & {$\left[0,10^{-1}\right]$} & Estimated \\
\hline$\alpha_{4}$ & $\begin{array}{l}\text { Rate of messaging by } \\
\text { infected symptomatic } \\
\text { individuals }\end{array}$ & {$\left[0,10^{-1}\right]$} & Estimated \\
\hline$\alpha_{5}$ & $\begin{array}{l}\text { Rate of messaging by } \\
\text { recovered individuals }\end{array}$ & {$\left[0,10^{-1}\right]$} & Estimated \\
\hline$\omega$ & $\begin{array}{l}\text { Outdating rate of media } \\
\text { campaigns }\end{array}$ & {$[0.2,0.5]$} & [17] \\
\hline
\end{tabular}
forward sensitivity index of a variable to a parameter is the ratio of the relative change in the variable to the relative change in the parameter. When the variable is a differentiable function of the parameter, the sensitivity index may be alternatively defined using partial derivatives (see [25]).
TABLE 2: Parameter values and their description.

Definition 6. The normalized forward sensitivity index of a variable, $u$, that depends differentiably on a parameter, $p$, is defined as

$$
\Upsilon_{p}^{u}:=\frac{\partial u}{\partial p} \times \frac{p}{u} .
$$

Media campaigns in this paper contribute to the limitation of the disease transmission. The reproduction number $R_{M}$ is an important concept when it comes to the disease transmission, because it helps to determine EVD incidence. The normalized forward sensitivity indices of $R_{M}$ with respect to each parameter $u$ in expression (22) are given by

$$
\Upsilon_{u}^{R_{M}}:=\frac{\partial R_{M}}{\partial u} \times \frac{u}{R_{M}} .
$$

Table 3 represents the numerical values of the sensitivity indices of the reproduction number $R_{M}$ for the parameters used in the model. The most important parameters are 
TABLE 3: Sensitivity indices for EVD reproduction number.

\begin{tabular}{lc}
\hline Parameter & Sensitivity index \\
\hline$\omega$ & +1.5 \\
$\mu$ & +1.37 \\
$\beta$ & +1 \\
$c$ & +1 \\
$\eta$ & +0.613 \\
$\gamma$ & +0.074 \\
$\theta$ & +0.037 \\
$\Lambda$ & -1.5 \\
$\alpha_{1}$ & -1.5 \\
$\rho$ & -0.613 \\
$\sigma$ & -0.347 \\
$p$ & -0.05 \\
$\delta_{1}$ & -0.022 \\
$\delta_{2}$ & -0.002 \\
\hline
\end{tabular}

those with the highest absolute values. Negative and positive correlations of the parameters to the reproduction number are indicated by negative and positive signs. The parameters $\Lambda$ and $\alpha_{1}$ have the largest absolute negative numerical values with negative sensitivity index values. Thus, increasing their values will decrease EVD incidence. This result can be explained by the fact that any increase in the two parameters leads to an increase in the efficacy of media campaigns. So, the more the media campaigns are efficacious, the less the value of the reproduction number is. Another important parameter with a negative index is $\rho$, which is an expected result since burials of EVD dead bodies limit the disease transmission due to infected corpses. The outdating rate of media campaigns $\omega$ has the most positive influence on EVD reproduction number. This means that the more frequently the messages spread by media on EVD are updated, the lower the number of new infections is. The larger the values of $\omega$ and $\mu$ are, the less the efficacy of the messages is and the more the disease spreads. The parameters $\beta$ and $c$ form the transmission rate and their increase will directly contribute to an increase in the number of EVD cases.

4.3. Simulations Results and Interpretation. Figures 5(a) and 5(b) confirm the results on stability analysis. It follows that when $R_{M}<1$, the epidemic dies out and, for $R_{M}>1$, EVD becomes endemic. This is a graphical description of the fact that the DFE is locally stable for $R_{M}<1$ and the EE is locally stable whenever $R_{M}>1$.

The media campaigns reproduction number $R_{M}$ is made of parameters which differently influence its values in a variety of ways. The relationship between those parameters can be evaluated through contour plots. We chose two parameters, $\alpha_{1}$ and $\omega$, whose influence on the reproduction number is clearly significant as shown in the expression of $R_{M}$. Figure 6 shows that $\alpha_{1}$ largely influences $R_{M}$ when compared to $\omega$. Increasing the values of $\alpha_{1}$ decreases $R_{M}$. Thus, in order to eradicate EVD, the exchange of EVD related messages is critical in eradicating the epidemic.

\section{Discussion and Conclusion}

To model the potential effect of media campaigns on Ebola transmission, we used a deterministic model, with compartments comprising individuals with different EVD infection status, who send EVD related messages through media. The effect of media campaigns on people's behaviour is represented by a reduction factor which decreases the number of new EVD cases. Stability analysis was presented in terms of the model reproduction number $R_{M}$. It was shown that the disease-free and the endemic equilibria are locally stable if $R_{M}<1$ and $R_{M}>1$, respectively. The inclusion of the asymptomatic infected class resulted in the model exhibiting a backward bifurcation, emphasizing the necessity of intense efforts against EVD as a result of undetected asymptomatic cases. The existence of a backward bifurcation has important implications in the design of policies and strategies to eradicate or control an epidemic. In the presence of a backward bifurcation, classical policies on disease eradication need to be changed as EVD can persist even when the threshold parameter $R_{M}$ is less than one.

To be able to control EVD, governments and international stakeholders should implement feasible campaigns taking into account the social, economic, and mainly the cultural realities of the affected countries. Interventions from these campaigns should target the affected populations and help them to understand the disease, comply with control measures, which sometimes seem severe, and change their behaviour in order to stop the disease transmission chain [1]. The best way to contain this outbreak is to jointly implement case isolation, contact tracing with quarantine, and sanitary funeral practices as suggested in [26].

This model is not without shortcomings. People's reaction to media campaigns does not always lead to a reduction in the number of future contamination cases. So, a function representing the influence of media campaigns on individuals' behaviour which takes into account the different cultural settings would be an innovative and informative addition to this model. Aspects of quarantine, contact tracing, and case identification initiatives are possible additions that can make this model more reliable. Despite these shortcomings, this model provides a good description of EVD outbreak. The model investigates a very important aspect in disease control in our times, that is, the use of social media in spreading messages.

\section{Appendix}

\section{A. Differential Inequalities}

Corollary A.1. Let $x_{0}$ and $y_{0}$ be real numbers, $I=\left[x_{0},+\infty\right)$, and $a, b \in C(I)$. Suppose that $y \in C^{1}(I)$ satisfies the following inequality:

$$
\begin{aligned}
& y^{\prime}(x) \leq a(x) y(x)+b(x), \quad x \geq x_{0}, y\left(x_{0}\right)=y_{0} . \\
& \text { Then, } \\
& y(x) \leq y_{0} \exp \left[\int_{x_{0}}^{x} a(t) d t\right] \\
& \quad+\int_{x_{0}}^{x} b(s) \exp \left[\int_{s}^{x} a(t) d t\right] d s, \quad x \geq x_{0} .
\end{aligned}
$$




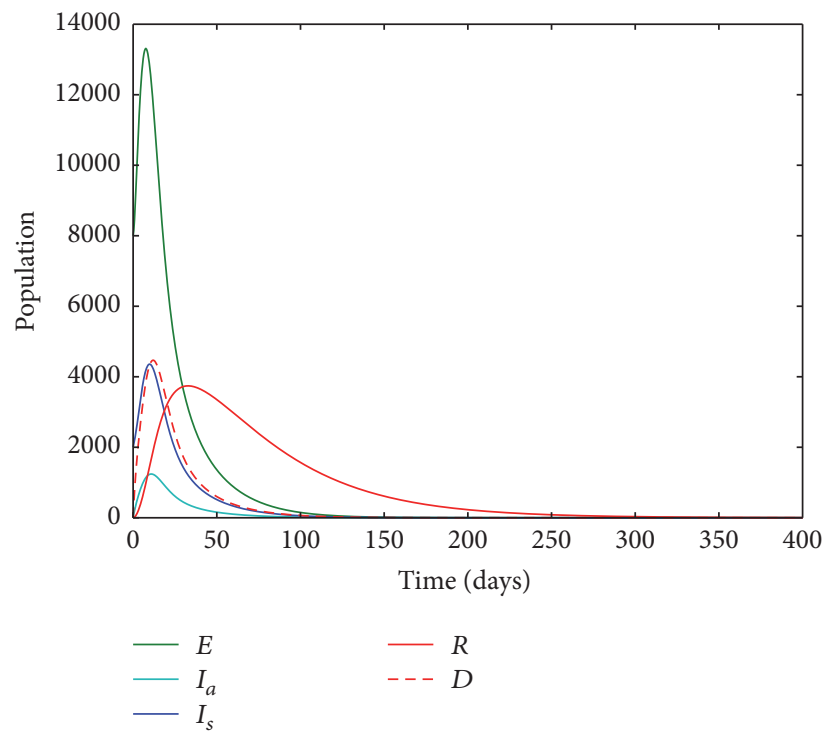

(a)

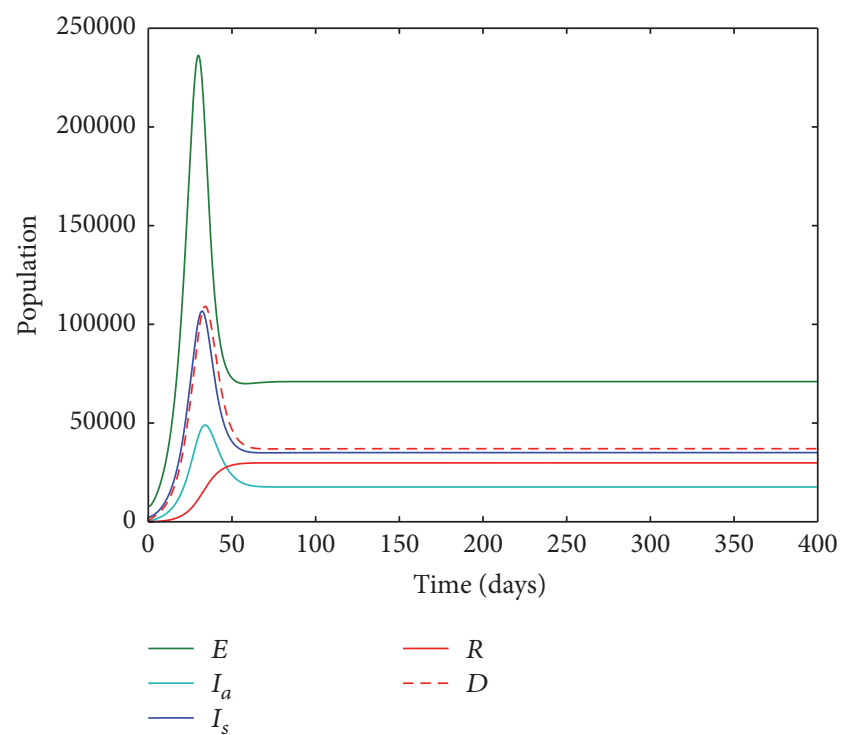

(b)

Figure 5: Population size at DFE (a) for $R_{M}=0.61$ and EE (b) for $R_{M}=2.09$ with $\Lambda=20000, \mu=0.02, \beta=(0.2,0.105), c=(8,12), \sigma=0.525$, $\gamma=(0.2,0.25), p=0.17, \theta=0.12, \delta_{1}=(0.199,0.0313), \delta_{2}=(0.0001,0.0013), \omega=0.2, \alpha_{1}=\left(1.8 \times 10^{-6}, 1.2 \times 10^{-7}\right), \alpha_{2}=\left(2 \times 10^{-7}, 2 \times 10^{-9}\right)$, $\alpha_{3}=\left(5 \times 10^{-6}, 5 \times 10^{-8}\right), \alpha_{4}=\left(8 \times 10^{-7}, 8 \times 10^{-8}\right), \alpha_{5}=\left(9.99 \times 10^{-7}, 9.9 \times 10^{-8}\right), \eta=1.5$, and $\rho=0.497$.

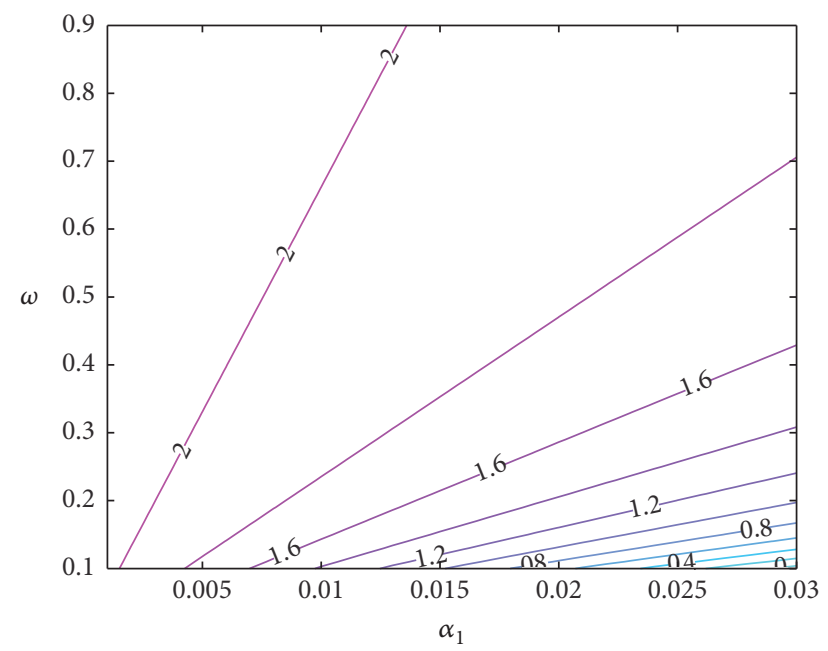

FIGURE 6: Reproduction number contour plot.

If the converse inequality holds in (A.1), then the converse inequality holds in (A.2) too.

Let us prove that $M(t)$ is bounded.

Proof. From (9), we have

$$
\frac{d M(t)}{d t} \geq \alpha_{1} S(t)-\omega M(t)
$$

For system (3)-(9), $M^{\prime}(t) \geq \alpha_{1} S-\omega M$. By applying Corollary A.1, we have

$$
\begin{aligned}
M(t) \geq & M(0) \exp \left[\int_{0}^{t}(-\omega) d u\right] \\
& +\int_{0}^{t} \alpha_{1} S \exp \left[\int_{z}^{t}(-\omega) d v\right] d z, \quad \forall t \geq 0,
\end{aligned}
$$

which yields

$$
M(t) \geq \exp [-\omega t]\left(M(0)-\frac{\alpha_{1} S}{\omega}\right)+\frac{\alpha_{1} S}{\omega} .
$$

Before the disease is spread, we assume that $M$ is at the steady state level. So, $M(0)=\alpha_{1} S / \omega$ which is equivalent to $M(0)=$ $M^{*}$ and (A.5) will give

$$
M(t) \geq \frac{\alpha_{1} S}{\omega} .
$$

Together with the assumption $0<M \leq 1$, we thus have

$$
M^{*} \leq M(t) \leq 1 \text {. }
$$

\section{B. An Approach to Determine the Direction of the Bifurcation}

Theorem B.1. Consider a general system of ordinary differential equations with a parameter $\phi$ :

$$
\frac{d x}{d t}=f(x, \phi)
$$

$$
f: R^{n} \times R \longrightarrow R^{n}, f \in C^{2}\left(R^{n} \times R\right) .
$$

Without loss of generality, it is assumed that 0 is an equilibrium for system (B.1) for all values of the parameter $\phi$; that is, $f(0, \phi) \equiv 0$ for all $\phi$.

Assume that

(A1) $A=D_{x} f(0,0)=\left(\left(\partial f_{i} / \partial x_{j}\right)(0,0)\right)$ is the linearisation matrix of system (B.1) around equilibrium 0 with $\phi$ evaluated at 0 . Zero is a simple eigenvalue of $A$ and all other eigenvalues of $A$ have negative real parts; 
(A2) matrix $A$ has a nonnegative right eigenvector $w$ and a left eigenvector $v$ corresponding to the zero eigenvalue. Let $f_{k}$ be the $k$ th component of $f$ and

$$
\begin{aligned}
& a=\sum_{k, i, j=1}^{n} v_{k} w_{i} w_{j} \frac{\partial^{2} f_{k}}{\partial x_{i} \partial x_{j}}(0,0), \\
& b=\sum_{k, i=1}^{n} v_{k} w_{i} \frac{\partial^{2} f_{k}}{\partial x_{i} \partial \phi}(0,0) .
\end{aligned}
$$

The local dynamics of (B.1) around 0 are totally determined by $a$ and $b$.

(1) $a>0, b>0$. When $\phi<0$ with $|\phi| \ll 1$, 0 is locally asymptotically stable, and there exists a positive unstable equilibrium; when $0<\phi \ll 1$, 0 is unstable and there exists a negative and locally asymptotically stable equilibrium.

(2) $a<0, b<0$. When $\phi<0$ with $|\phi| \ll 1,0$ is unstable; when $0<\phi=1,0$ is locally asymptotically stable, and there exists a positive unstable equilibrium.

(3) $a>0, b<0$. When $\phi<0$ with $|\phi| \ll 1,0$ is unstable, and there exists a locally asymptotically stable negative equilibrium; when $0<\phi \ll 1,0$ is stable, and a positive unstable equilibrium appears.

(4) $a<0, b>0$. When $\phi$ changes from negative to positive, 0 changes its stability from stable to unstable. Correspondingly, a negative unstable equilibrium becomes positive and locally asymptotically stable.

Corollary B.2. When $a>0$ and $b>0$, the bifurcation at $\phi=0$ is subcritical or backward.

\section{Competing Interests}

The authors declare that there are no competing interests regarding the publication of this paper.

\section{Authors' Contributions}

Farai Nyabadza developed the model and supervised Sylvie Diane Djiomba Njankou during her M.S. which resulted in this paper. Sylvie Diane Djiomba Njankou carried out the analysis and model simulations.

\section{Acknowledgments}

This publication has benefited from the intellectual and material contribution of the Organization for Women in Science for the Developing World (OWSD) and the Swedish International Development Cooperation Agency (SIDA). The second author acknowledges the support of Stellenbosch University in the production of this manuscript.

\section{References}

[1] World Health Organisation, Ebola and Marburg virus disease epidemics: preparedness, alert, control, and evaluation, WHO/HSE/PED/CED/2014.05, 2014.

[2] Centers for Disease Control and Prevention, Morbidity and Mortality Weekly Report, https://www.cdc.gov/mmwr/preview/mmwrhtml/mm6325a4.htm.

[3] J. J. Muyembe-Tamfum, S. Mulangu, J. Masumu, J. M. Kayembe, A. Kemp, and J. T. Paweska, "Ebola virus outbreaks in Africa: past and present," Onderstepoort Journal of Veterinary Research, vol. 79, no. 2, 8 pages, 2012.

[4] E. M. Leroy, S. Baize, V. E. Volchkov et al., "Human asymptomatic Ebola infection and strong inflammatory response," The Lancet, vol. 355, no. 9222, pp. 2210-2215, 2000.

[5] J. Astacio, D. Briere, M. Guillen, J. Martinez, F. Rodriguez, and N. Valuenzuela-Campos, "Mathematical Models to study the outbreaks of Ebola," http://ecommons.cornell.edu/bitstream/ 1813/31962/1/BU-1365-M.pdf.

[6] C. N. Haas, "On the quarantine period for ebola virus," PLoS Currents Outbreaks, 2014.

[7] S. E. Bellan, J. R. C. Pulliam, J. Dushoff, and L. A. Meyers, "Ebola control: effect of asymptomatic infection and acquired immunity," The Lancet, vol. 384, no. 9953, pp. 1499-1500, 2014.

[8] A. G. Baxter, "Symptomeless infection with Ebola virus," Commentary, vol. 355, article 2210, 2014.

[9] World Health Organisation, Case Definition Recommendations for Ebola or Marburg Virus Diseases, WHO, Geneva, Switzerland, 2014.

[10] Y. Xiao, T. Zhao, and S. Tang, "Dynamics of an infectious diseases with media/psychology induced non-smooth incidence," Mathematical Biosciences and Engineering, vol. 10, no. 2, pp. 445-461, 2013.

[11] Y. Xiao, S. Tang, and J. Wu, "Media impact switching surface during an infectious disease outbreak," Scientific Reports, vol. 5, article 7838, 2015.

[12] J. M. Tchuenche and C. T. Bauch, "Dynamics of an infectious disease where media coverage influences transmission," ISRN Biomathematics, vol. 2012, Article ID 581274, 10 pages, 2012.

[13] A. Sharma and A. K. Misra, "Backward bifurcation in a smoking cessation model with media campaigns," Applied Mathematical Modelling, vol. 39, no. 3-4, pp. 1087-1098, 2015.

[14] K. A. Pawelek, A. Oeldorf-Hirsch, and L. Rong, "Modeling the impact of Twitter on influenza epidemics," Mathematical Biosciences and Engineering. MBE, vol. 11, no. 6, pp. 1337-1356, 2014.

[15] S. D. Djiomba Njankou and F. Nyabadza, Modelling the potential role of control strategies on Ebola virus disease dynamics (Master thesis in Mathematical Biology) [M.S. thesis], Stellenbosch University, Stellenbosch, South Africa, 2015.

[16] C. M. Rivers, E. T. Lofgren, M. Marathe, S. Eubank, and B. L. Lewis, "Modelling the impact of interventions on an epidemic of Ebola in Sierra Leone and Liberia," PLOS Current Outbreaks, vol. 6, 2014.

[17] Centers for Disease Control and Prevention: 2014 Ebola outbreak in West Africa, http://www.cdc.gov/vhf/ebola/outbreaks/ 2014-west-africa/case-counts.html.

[18] S. L. Coyle, R. F. Boruch, and C. F. Turner, Evaluating AIDS Prevention Programs: Expanded Edition, National Academies Press, Washington, DC, USA, 1991. 
[19] P. van den Driessche and J. Watmough, "Reproduction numbers and sub-threshold endemic equilibria for compartmental models of disease transmission," Mathematical Biosciences, vol. 180, no. 1-2, pp. 29-48, 2002.

[20] J. P. LaSalle and Z. Artstein, The Stability of Dynamical Systems: Appendix A Limiting Equations and Stability of non Autonomous Ordinary Differential Equations, Society for Industrial and Applied Mathematics, 1876.

[21] K. P. Hadeler and P. Van Den Driessche, Backward Bifurcation in Epidemic Control, Elsevier, 1997.

[22] C. Castillo-Chavez and B. Song, "Dynamical models of tuberculosis and their applications," Mathematical Biosciences and Engineering, vol. 1, no. 2, pp. 361-404, 2004.

[23] Geonames, http://www.geonames.org/8335091/nzerekore.html.

[24] D. G. Cacuci, Sensitivity and Uncertainty Analysis, vol. 1, Chapman \& Hall/CRC, 2003.

[25] N. Chitnis, J. M. Hyman, and J. M. Cushing, "Determining important parameters in the spread of malaria through the sensitivity analysis of a mathematical model," Bulletin of Mathematical Biology, vol. 70, no. 5, pp. 1272-1296, 2008.

[26] A. Pandey, K. E. Atkins, J. Medlock et al., "Strategies for containing Ebola in West Africa," Science, vol. 346, no. 6212, pp. 991-995, 2014. 


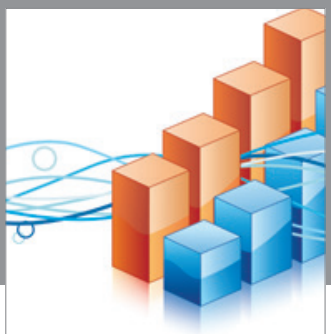

Advances in

Operations Research

vatem alat4

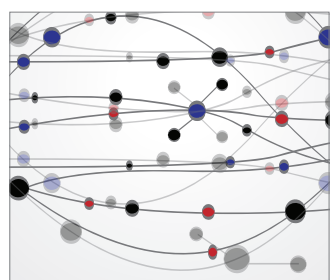

\section{The Scientific} World Journal
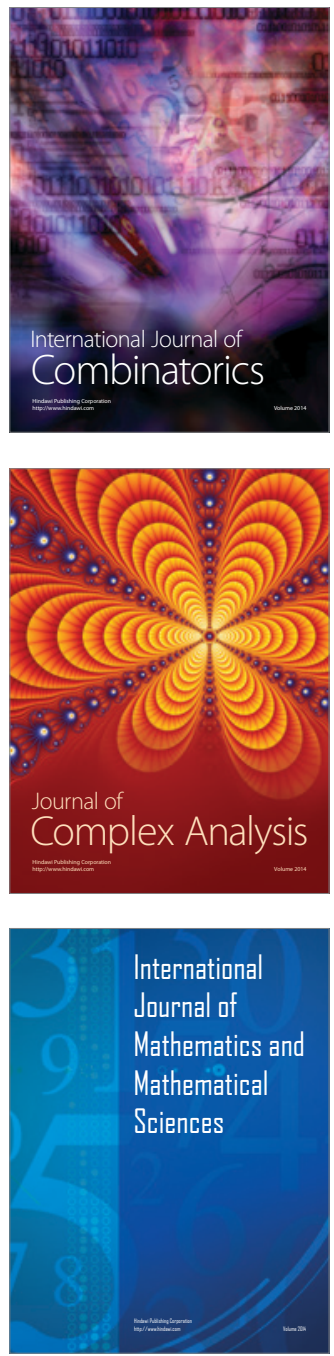
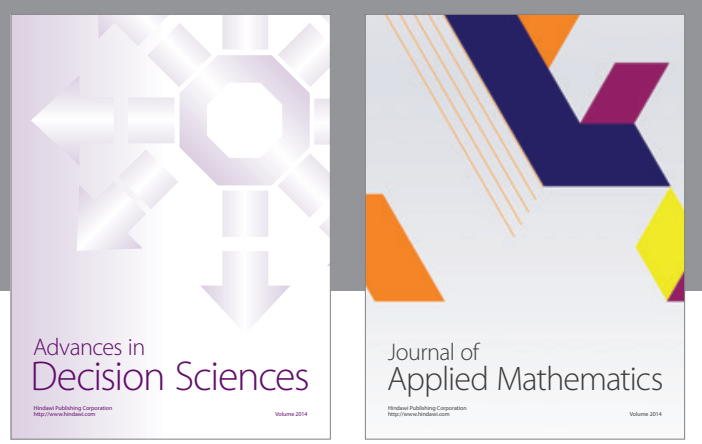

Algebra

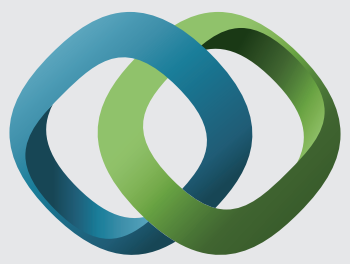

\section{Hindawi}

Submit your manuscripts at

https://www.hindawi.com
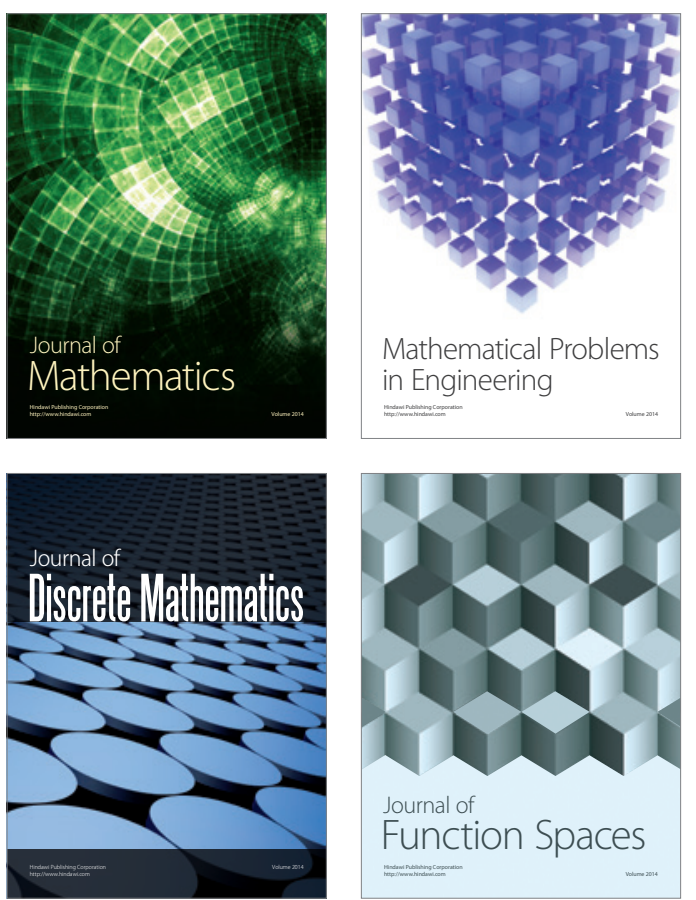

Mathematical Problems in Engineering
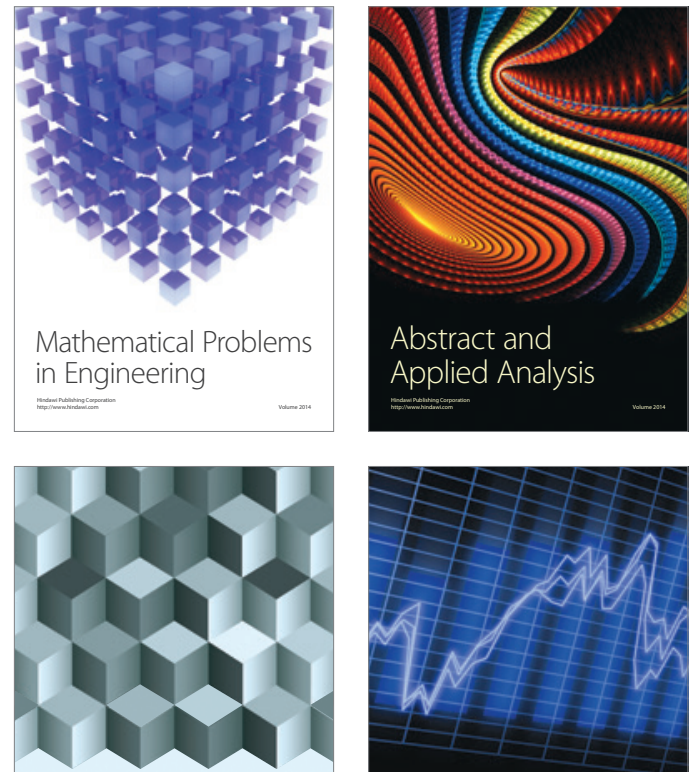

Journal of

Function Spaces

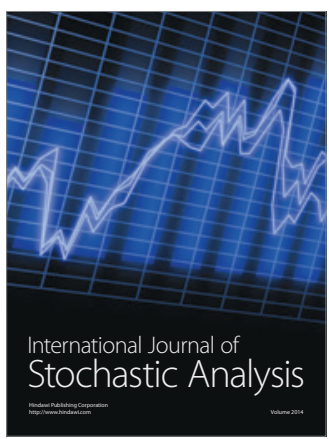

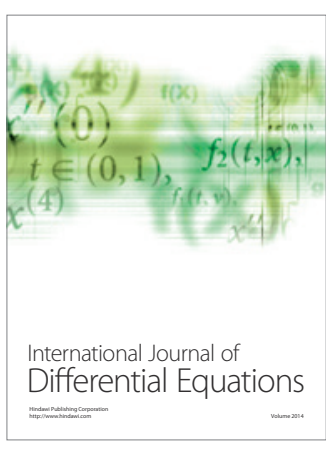
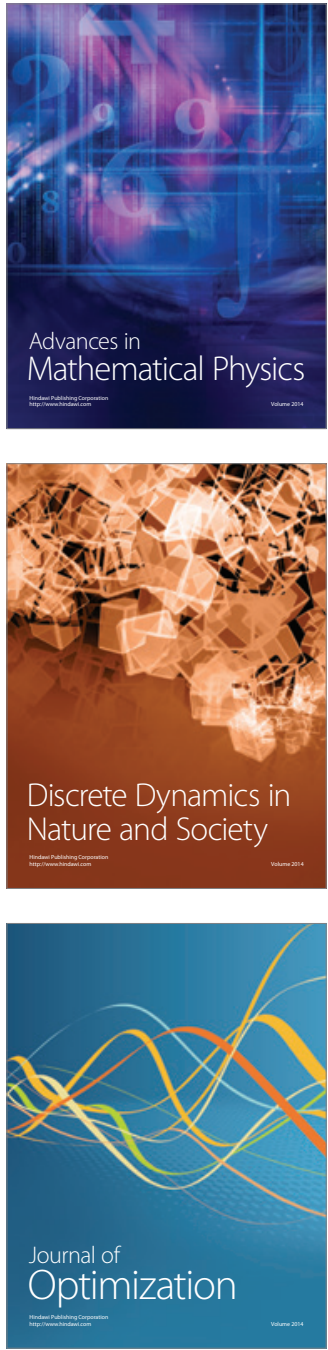